\title{
Ceftolozane/Tazobactam for Resistant Drugs Pseudomonas aeruginosa Respiratory Infections: A Systematic Literature Review of the Real-World Evidence
}

\author{
Luca Gregorio Giaccari ${ }^{1}$, Maria Caterina Pace ${ }^{1}$, Maria Beatrice Passavanti ${ }^{1}$ (D), Francesca Gargano ${ }^{2}$, \\ Caterina Aurilio ${ }^{1}$ and Pasquale Sansone ${ }^{1, *(D)}$
}

1 Department of Women, Child, General and Specialistic Surgery, University of Campania "L. Vanvitelli", 80138 Naples, Italy; lucagregorio.giaccari@gmail.com (L.G.G.); mariacaterina.pace@unicampania.it (M.C.P.); mariabeatrice.passavanti@unicampania.it (M.B.P.); Caterina.aurilio@unicampania.it (C.A.)

2 Unit of Anaesthesia, Intensive Care and Pain Management, Università Campus Bio-Medico di Roma, 00128 Rome, Italy; f.gargano@unicampus.it

* Correspondence: pasquale.sansone@unicampania.it

Citation: Giaccari, L.G.; Pace, M.C.; Passavanti, M.B.; Gargano, F.; Aurilio, C.; Sansone, P. Ceftolozane/Tazobactam for Resistant Drugs Pseudomonas aeruginosa Respiratory Infections: A Systematic Literature Review of the Real-World Evidence. Life 2021, 11, 474. https://doi.org/10.3390/ life11060474

Academic Editor: Payaningal R. Somanath

Received: 17 April 2021

Accepted: 20 May 2021

Published: 24 May 2021

Publisher's Note: MDPI stays neutral with regard to jurisdictional claims in published maps and institutional affiliations.

Copyright: (C) 2021 by the authors Licensee MDPI, Basel, Switzerland. This article is an open access article distributed under the terms and conditions of the Creative Commons Attribution (CC BY) license (https:// creativecommons.org/licenses/by/ $4.0 /)$.

\begin{abstract}
Background: Ceftolozane/tazobactam (C/T) is a $\beta$-lactam/ $\beta$-lactamase inhibitor combination that mainly targets Gram-negative bacteria. The current international guidelines recommend including $\mathrm{C} / \mathrm{T}$ treatment in the empirical therapy for hospital-acquired pneumonia (HAP) and ventilator-associated pneumonia (VAP). Pseudomonas aeruginosa (PA) is one of the most challenging Gram-negative bacteria. We conducted a systematic review of all cases reported in the literature to summarize the existing evidence. Methods: The main electronic databases were screened to identify case reports of patients with drug-resistant PA respiratory infections treated with C/T. Results: A total of 22 publications were included for a total of 84 infective episodes. The clinical success rate was $72.6 \%$ across a wide range of comorbidities. The $45.8 \%$ of patients treated with $\mathrm{C} / \mathrm{T}$ presented colonization by PA. C/T was well tolerated. Only six patients presented adverse events, but none had to stop treatment. The most common therapeutic regimens were $1.5 \mathrm{~g}$ every $8 \mathrm{~h}$ and $3 \mathrm{~g}$ every $8 \mathrm{~h}$. Conclusion: C/T may be a valid therapeutic option to treat multidrug-resistant (MDR), extensively drug-resistant (XDR), pandrug-resistant (PDR), and carbapenem-resistant (CR) PA infections. However, further data are necessary to define the optimal treatment dosage and duration.
\end{abstract}

Keywords: ceftolozane/tazobactam; hospital-acquired pneumonia (HAP); ventilator-associated pneumonia (VAP); Pseudomonas aeruginosa; multidrug-resistant (MDR); extensively drug-resistant (XDR); pandrug-resistant (PDR); carbapenem-resistant (CR)

\section{Introduction}

Ceftolozane/tazobactam $(\mathrm{C} / \mathrm{T})$ is a $\beta$-lactam/ $\beta$-lactamase inhibitor combination that mainly targets Gram-negative bacteria [1]. The U.S. Food and Drug Administration (FDA) in 2014 and then the European Medicines Agency (EMA) in 2015 approved the use of $\mathrm{C} / \mathrm{T}[2,3]$. Originally, $\mathrm{C} / \mathrm{T}$ was indicated for the treatment of complicated intra-abdominal infections (cIAIs) and complicated urinary tract infections (cUTIs) caused by the following Gram-negative and Gram-positive microorganisms: Enterobacter cloacae, Escherichia coli, Klebsiella oxytoca, Klebsiella pneumoniae, Proteus mirabilis, Pseudomonas aeruginosa, Bacteroides fragilis, Streptococcus anginosus, Streptococcus constellatus, and Streptococcus salivarius [4,5]. The recommended dosage regimen of $C / T$ was $1.5 \mathrm{~g}$ administered every $8 \mathrm{~h}$.

$\mathrm{C} / \mathrm{T}$ was soon investigated as a possible therapeutic agent for nosocomial pneumonia due to its potent activity against Pseudomonas aeruginosa (PA) [6], responsible for a large proportion of hospital-acquired pneumonia (HAP) and ventilator-associated pneumonia (VAP) $[7,8]$. The current international guidelines recommend including $\mathrm{C} / \mathrm{T}$ treatment in the empirical therapy for HAP and VAP $[9,10]$. 
In 2019, the FDA and EMA approved C/T as a therapeutic option for treating HAP and VAP [11,12]. The recommended dosage regimen of $\mathrm{C} / \mathrm{T}$ was $3 \mathrm{~g}$ three times a day (t.i.d.) by intermittent infusion.

PA is one of the most challenging Gram-negative bacteria due to its intrinsic and acquired resistance mechanisms such as its ability to develop biofilms [13]. PA exhibits multidrug-resistant (MDR), extensively drug-resistant (XDR), and pandrug-resistant (PDR) phenotypes, reducing the number of antimicrobial agents available for clinical use [14]. In 2018, the World Health Organization (WHO) selected carbapenem-resistant (CR) PA as a critical-priority bacterium for future development strategies focused on new antibiotics [15].

In this scenario, $\mathrm{C} / \mathrm{T}$ could play a central role. Ceftolozane is a cephalosporin with potent in vitro activity against PA that is not influenced by the $\beta$-lactam resistance mechanisms present in this species [16]. The combination with the tazobactam, a $\beta$-lactamase inhibitor, extends ceftolozane activity against many Enterobacteriaceae producing ESBLs $[17,18]$. According to the Italian survey on PA, C/T was the most active anti-PA agent and, moreover, it was active against approximately half the isolates that are resistant to all other $\beta$-lactams or resistant to all other agents except colistin [14].

Objectives. We conducted a systematic review of all cases reported in the literature to summarize the existing evidence. To our knowledge, no previously published systematic reviews have evaluated real-world evidence studies of $\mathrm{C} / \mathrm{T}$ in the treatment of PA resistant to multiple antimicrobial agents. Although a systematic review of case reports cannot support efficacy between drug-resistant PA respiratory infections and $\mathrm{C} / \mathrm{T}$ therapy, it may identify unrecognized or rare associations and may generate hypotheses for subsequent studies.

\section{Materials and Methods}

\subsection{Protocol and Registration}

A systematic review was performed on the basis of the PRISMA (Preferred Reporting Items for Systematic Reviews and Meta-Analyses) guidelines [19]. The protocol was not published, but is available on request. The review was not registered with the international prospective register of systematic reviews (PROSPERO) due to the long waiting times for the current situation.

\subsection{Literature Search Strategy}

The main electronic databases (Medline, EMBASE, PubMed, Google Scholar, and The Cochrane Library-CENTRAL) were screened to identify case reports of patients with drugresistant PA respiratory infections treated with $\mathrm{C} / \mathrm{T}$. Other relevant studies were identified from the reference lists. We used a combination of terms such as "ceftolozane-tazobactam", "Pseudomonas aeruginosa", "drug resistance", and "pneumonia". The titles and abstracts were screened by two researchers (L.G.G. and M.C.P.) to identify the keywords. The selected papers were read in full by the two independent reviewers, and a third reviewer (P.S.) was consulted in cases of disagreement.

The initial search was performed on 1 April 2021. All publications were included since inception up until the end of March 2021.

All the papers with available full text, reporting original data of patients with drugresistant PA respiratory infections treated with $\mathrm{C} / \mathrm{T}$, of any age, gender, and in any setting, were included. No language restrictions were applied.

\subsection{Inclusion and Exclusion Criteria}

Studies were included if they met all of the following criteria:

- the full study was published;

- the study described clinical use of C/T for respiratory infections;

- the responsible agent of the infection was PA resistant to multiple antimicrobial agents;

- the study reported the clinical outcome of the patient(s) treated with $\mathrm{C} / \mathrm{T}$.

Reasons for exclusions were: 
- the study did not report clinical outcome;

- the study had duplicate data with others (in these cases, only the largest study was retained);

- the study presented pooled data that did not allow for extrapolation of useful information.

\subsection{Data Extraction}

Data were independently extracted by one of the three reviewers (L.G.G., M.C.P., P.S.) according to a predefined protocol. The data extraction was then checked by one of the other two reviewers, and the discrepancies were resolved by discussion between all of them.

Variables of interest included:

- demographic characteristics (sex and age);

- clinical characteristics (commodities);

- type of infection and resistance profile;

- therapeutic regimen (empirical and targeted) and dosage;

- co-infections;

- adverse events (AEs);

- clinical and microbiological outcome.

According to an international expert proposal, MDR is defined as "non-susceptibility to at least one agent in three or more antimicrobial categories", XDR is defined as "nonsusceptibility to at least one agent in all but two or fewer antimicrobial categories (i.e., bacterial isolates remain susceptible to only one or two categories)", and PDR is defined as "non-susceptibility to all agents in all antimicrobial categories (i.e., no agents tested as susceptible for that organism)" [20].

\section{Results}

A total of 22 full texts were eligible for inclusion, namely, 4 cohort studies and 18 case reports/case series investigating 84 infective episodes in total. As shown in Figure 1, the flow diagram reports the results from the literature search and the study selection process.

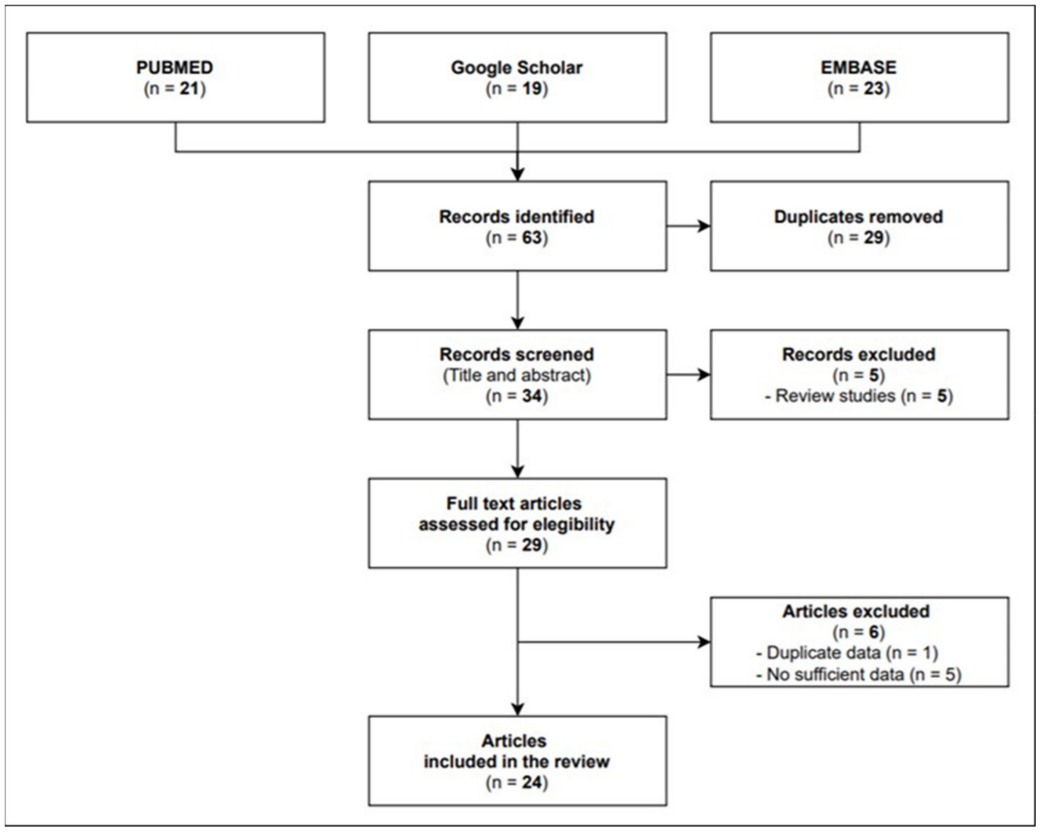

Figure 1. Flow diagram study selection process.

\section{Study Characteristics}

In Table 1, all the studies are presented in alphabetical order with a brief clinical description for each case. 
Table 1. Case reports of PA pulmonary infection.

\begin{tabular}{|c|c|c|c|c|c|c|c|c|}
\hline Author, Year & Study Design & No. & Sex, Years & Type of Infection & Resistance Profile & $\begin{array}{c}\text { C/T Dosage } \\
\text { (Duration, Days) }\end{array}$ & $\begin{array}{l}\text { Clinical } \\
\text { Outcome }\end{array}$ & $\begin{array}{c}\text { Microbiological } \\
\text { Status }\end{array}$ \\
\hline Alessa et al., 2018 [21] & Case report & 1 & M, 79 & $\begin{array}{l}\text { Aspiration } \\
\text { pneumonia }\end{array}$ & MDR & $\begin{array}{c}1.5 \mathrm{~g} \rightarrow \\
300 \mathrm{mg} / 8 \mathrm{~h}(13)\end{array}$ & S & $\mathrm{N} / \mathrm{D}$ \\
\hline Alqaid et al., 2015 [22] & Case report & 1 & M, 69 & VAP & PDR & $1.5 \mathrm{~g} / 8 \mathrm{~h} \mathrm{(14)}$ & $\mathrm{S}$ & $\mathrm{N} / \mathrm{D}$ \\
\hline \multirow[t]{2}{*}{ Álvarez Lerma et al., 2017 [23] } & \multirow[t]{2}{*}{ Case report } & \multirow[t]{2}{*}{2} & M, 72 & VAP & PDR & $\begin{array}{l}1.5 \mathrm{~g} / 8 \mathrm{~h}(4) \rightarrow \\
750 \mathrm{mg} / 8 \mathrm{~h}(10)\end{array}$ & $\mathrm{S}$ & $\mathrm{C}$ \\
\hline & & & $\mathrm{M}, 48$ & VAP & PDR & $750 \mathrm{mg} / 8 \mathrm{~h} \mathrm{(17)}$ & $S$ & $\mathrm{C}$ \\
\hline \multirow[t]{3}{*}{ Ang et al., 2016 [24] } & \multirow[t]{2}{*}{ Case report } & \multirow[t]{2}{*}{1} & $\mathrm{~F}, 14$ & Pneumonia & MDR & $\begin{array}{c}1.5 \mathrm{~g} / 8 \mathrm{~h} \rightarrow 750 \\
\mathrm{mg} / 8 \mathrm{~h} \mathrm{(14)}\end{array}$ & $\mathrm{S}$ & $\mathrm{E}$ \\
\hline & & & $\mathrm{F}, 45$ & Pneumonia & $\mathrm{CR}$ & $1.5 \mathrm{~g} / 8 \mathrm{~h}(7)$ & $\mathrm{F}$ & C \\
\hline & \multirow{5}{*}{$\begin{array}{l}\text { Observational } \\
\text { study }\end{array}$} & \multirow{5}{*}{6} & $?$ & Pneumonia & $\mathrm{CR}$ & ? & S & $\mathrm{N} / \mathrm{D}$ \\
\hline \multirow{4}{*}{ Bosaeed et al., 2020 [25] } & & & $?$ & Pneumonia & $\mathrm{CR}$ & $?$ & S & $\mathrm{N} / \mathrm{D}$ \\
\hline & & & M, 69 & VAP & $\mathrm{CR}$ & $1.5 \mathrm{~g} / 8 \mathrm{~h}(14)$ & $\mathrm{F}$ & C \\
\hline & & & M, 61 & VAP & $\mathrm{CR}$ & $1.5 \mathrm{~g} / 8 \mathrm{~h}(8)$ & $\mathrm{F}$ & C \\
\hline & & & $?$ & VAP & CR & ? & $S$ & $\mathrm{~N} / \mathrm{D}$ \\
\hline \multirow{5}{*}{ Castón et al., 2016 [26] } & \multirow{5}{*}{ Case series } & \multirow{5}{*}{6} & $\mathrm{~F}, 75$ & Pneumonia & MDR & $3 \mathrm{~g} / 8 \mathrm{~h}(10)$ & $S$ & E \\
\hline & & & M, 37 & Pneumonia & MDR & $3 \mathrm{~g} / 8 \mathrm{~h}(10)$ & S & E \\
\hline & & & M, 74 & Pneumonia & MDR & $3 \mathrm{~g} / 8 \mathrm{~h}(15)$ & F (death) & $\mathrm{E}$ \\
\hline & & & M, 61 & Pneumonia & MDR & $3 \mathrm{~g} / 8 \mathrm{~h} \mathrm{(3)}$ & F (death) & C \\
\hline & & & M, 58 & Pneumonia & MDR & $1.5 \mathrm{~g} / 8 \mathrm{~h}(21)$ & $\mathrm{S}$ & $\mathrm{C}$ \\
\hline \multirow{7}{*}{ Dinh et al., 2017 [27] } & \multirow{7}{*}{ Case series } & \multirow{7}{*}{7} & M, 61 & VAP & XDR & $3 \mathrm{~g} / 8 \mathrm{~h}(15)$ & $S$ & $\mathrm{E}$ \\
\hline & & & $\mathrm{F}, 70$ & VAP & XDR & $3 \mathrm{~g} / 8 \mathrm{~h}(10)$ & $\mathrm{S}$ & $\mathrm{C}$ \\
\hline & & & $\mathrm{M}, 60$ & VAP & XDR & $3 \mathrm{~g} / 8 \mathrm{~h}(15)$ & $\mathrm{S}$ & $\mathrm{C}$ \\
\hline & & & $\mathrm{F}, 73$ & VAP & XDR & $1.5 / 8 \mathrm{~h}(18)$ & $\mathrm{F}$ & E \\
\hline & & & M, 73 & VAP & XDR & $1.5 / 8 \mathrm{~h}(4)$ & $\mathrm{F}$ & $\mathrm{N} / \mathrm{D}$ \\
\hline & & & M, 49 & VAP & XDR & $3 \mathrm{~g} / 8 \mathrm{~h}(11)$ & S & $\mathrm{N} / \mathrm{D}$ \\
\hline & & & M, 38 & Pneumonia & XDR & $?(5)$ & S & $\mathrm{N} / \mathrm{D}$ \\
\hline
\end{tabular}


Table 1. Cont.

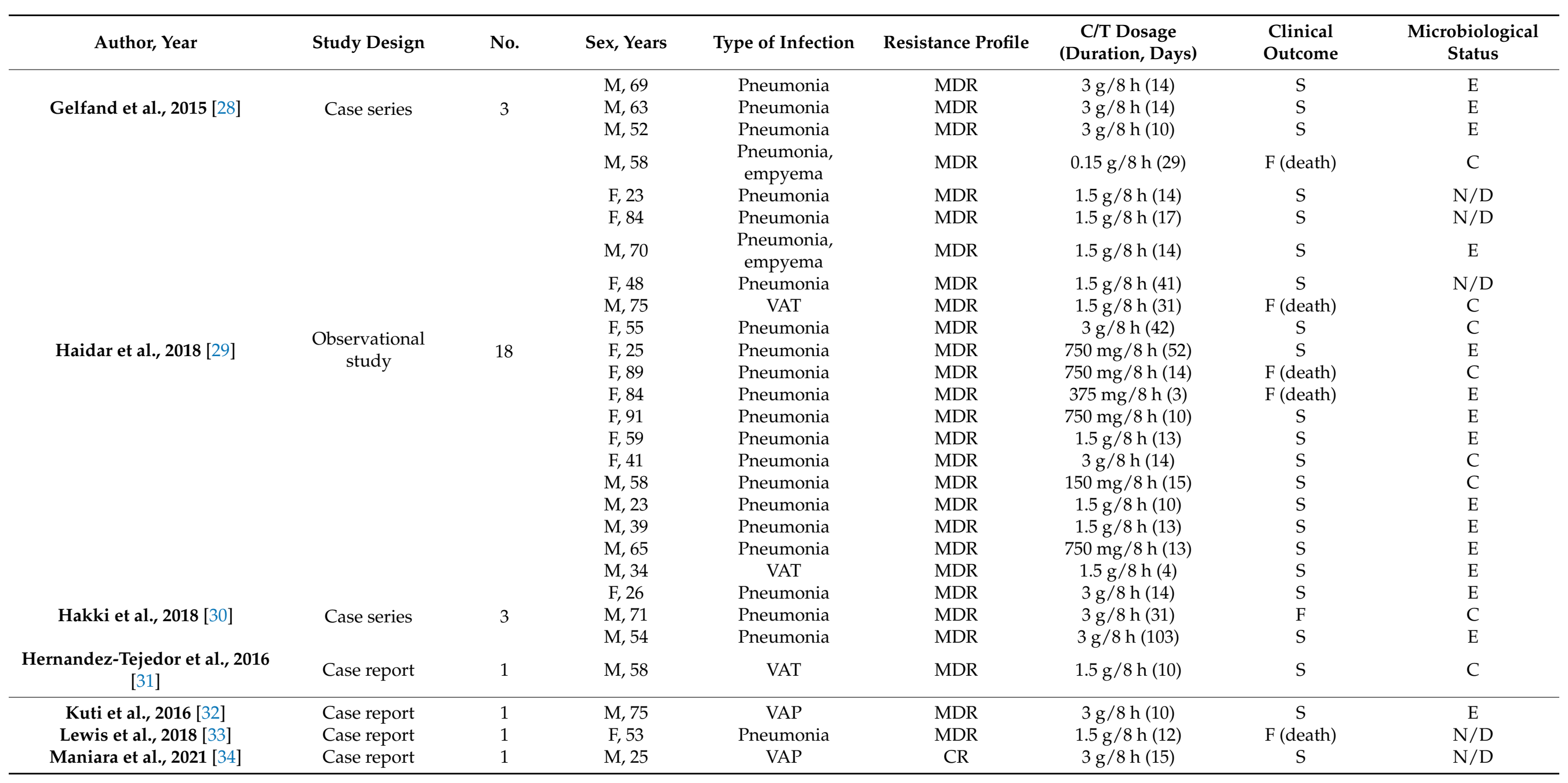


Table 1. Cont.

\begin{tabular}{|c|c|c|c|c|c|c|c|c|}
\hline Author, Year & Study Design & No. & Sex, Years & Type of Infection & Resistance Profile & $\begin{array}{c}\text { C/T Dosage } \\
\text { (Duration, Days) }\end{array}$ & $\begin{array}{l}\text { Clinical } \\
\text { Outcome }\end{array}$ & $\begin{array}{l}\text { Microbiological } \\
\text { Status }\end{array}$ \\
\hline \multirow{15}{*}{ Munita et al., 2017 [35] } & \multirow{15}{*}{$\begin{array}{l}\text { Observational } \\
\text { study }\end{array}$} & \multirow{15}{*}{18} & M, 31 & Pneumonia & $\mathrm{CR}$ & $1.5 \mathrm{~g} / 8 \mathrm{~h} \mathrm{(14)}$ & F (death) & $\mathrm{N} / \mathrm{D}$ \\
\hline & & & M, 38 & Pneumonia & $\mathrm{CR}$ & $750 \mathrm{mg} / 8 \mathrm{~h}(42)$ & $\mathrm{S}$ & $\mathrm{N} / \mathrm{D}$ \\
\hline & & & M, 32 & Pneumonia & $\mathrm{CR}$ & $1.5 \mathrm{~g} / 8 \mathrm{~h} \mathrm{(18)}$ & $\mathrm{F}$ & $\mathrm{N} / \mathrm{D}$ \\
\hline & & & M, 35 & Pneumonia & $\mathrm{CR}$ & $3 \mathrm{~g} / 8 \mathrm{~h}(9)$ & $S$ & $\mathrm{~N} / \mathrm{D}$ \\
\hline & & & M, 25 & Pneumonia & $\mathrm{CR}$ & $1.5 \mathrm{~g} / 8 \mathrm{~h}(8)$ & $S$ & $\mathrm{~N} / \mathrm{D}$ \\
\hline & & & M, 30 & Pneumonia & $\mathrm{CR}$ & $1.5 \mathrm{~g} / 8 \mathrm{~h}(14)$ & S & $\mathrm{N} / \mathrm{D}$ \\
\hline & & & M, 55 & Pneumonia & $\mathrm{CR}$ & $375 \mathrm{mg} / 8 \mathrm{~h}(12)$ & $\mathrm{F}$ & $\mathrm{N} / \mathrm{D}$ \\
\hline & & & M, 39 & Pneumonia & $\mathrm{CR}$ & $1.5 \mathrm{~g} / 8 \mathrm{~h}(7)$ & F (death) & C \\
\hline & & & M, 66 & Pneumonia & $\mathrm{CR}$ & $375 \mathrm{mg} / 8 \mathrm{~h} \mathrm{(16)}$ & $\mathrm{S}$ & $\mathrm{N} / \mathrm{D}$ \\
\hline & & & $\mathrm{F}, 84$ & Pneumonia & CR & 375 mg/8 h (8) & $S$ & $\mathrm{~N} / \mathrm{D}$ \\
\hline & & & M, 67 & Pneumonia & $\mathrm{CR}$ & 375 mg/8 h (14) & S & $\mathrm{N} / \mathrm{D}$ \\
\hline & & & M, 63 & Pneumonia & $\mathrm{CR}$ & $375 \mathrm{mg} / 12 \mathrm{~h}(16)$ & S & $\mathrm{N} / \mathrm{D}$ \\
\hline & & & M, 61 & Pneumonia & $\mathrm{CR}$ & $375 \mathrm{mg} / 8 \mathrm{~h}(5)$ & F (death) & $\mathrm{N} / \mathrm{D}$ \\
\hline & & & M, 71 & Pneumonia & CR & $3 \mathrm{~g} / 8 \mathrm{~h} \mathrm{(5)}$ & $\mathrm{F}$ & $\mathrm{N} / \mathrm{D}$ \\
\hline & & & $\mathrm{F}, 61$ & Pneumonia & $\mathrm{CR}$ & $3 \mathrm{~g} / 8 \mathrm{~h}(22)$ & $S$ & $\mathrm{~N} / \mathrm{D}$ \\
\hline Plant et al., 2018 [36] & Case report & 1 & $\mathrm{M}, ?$ & Pneumonia & MDR & $1.5 \mathrm{~g} / 8 \mathrm{~h}(?)$ & F (death) & C \\
\hline Romano et al., 2020 [37] & Case report & 1 & $\mathrm{~F}, 63$ & Pneumonia & MDR & $3 \mathrm{~g} / 8 \mathrm{~h}(14)$ & $\mathrm{S}$ & $\mathrm{N} / \mathrm{D}$ \\
\hline Soliman et al., 2015 [38] & Case report & 1 & M, 59 & Pneumonia & PDR & $3 \mathrm{~g} / 8 \mathrm{~h}(14)$ & S & $\mathrm{N} / \mathrm{D}$ \\
\hline Stokem et al., 2018 [39] & Case report & 1 & $\mathrm{~F}, 35$ & Pneumonia & MDR & $3 \mathrm{~g} / 12 \mathrm{~h}(14)$ & $S$ & C \\
\hline \multirow[t]{4}{*}{ Vickery et al., 2016 [40] } & Case report & 1 & M, 25 & Pneumonia & MDR & $3 \mathrm{~g} / 8 \mathrm{~h}(12)$ & $S$ & $\mathrm{C}$ \\
\hline & & & M, 78 & Pneumonia & MDR & ? & S & $\mathrm{E}$ \\
\hline & & & M, 69 & Pneumonia & XDR & $1.5 \mathrm{~g} / 8 \mathrm{~h}(6)$ & F (death) & C \\
\hline & & & $\mathrm{M}, 52$ & Pneumonia & MDR & $1.5 \mathrm{~g} / 8 \mathrm{~h} \mathrm{(8)}$ & S & $\mathrm{N} / \mathrm{D}$ \\
\hline \multirow[t]{5}{*}{ Xipell et al., 2018 [41] } & $\begin{array}{l}\text { Observational } \\
\text { study }\end{array}$ & 8 & M, 77 & Pneumonia & XDR & $\begin{array}{c}3 \mathrm{~g} / 8 \mathrm{~h}(3) \rightarrow 2 \\
\mathrm{~g} / 8 \mathrm{~h} \mathrm{(3)}\end{array}$ & $S$ & $\mathrm{~N} / \mathrm{D}$ \\
\hline & & & $\mathrm{F}, 49$ & Tracheobronchitis & XDR & $1.5 \mathrm{~g} / 8 \mathrm{~h}(8)$ & S & $\mathrm{E}$ \\
\hline & & & M, 49 & Tracheobronchitis & MDR & ? & $S$ & E \\
\hline & & & M, 64 & Tracheobronchitis & XDR & $1.5 \mathrm{~g} / 8 \mathrm{~h}(7)$ & $\mathrm{F}$ & $\mathrm{E}$ \\
\hline & & & M, 61 & Tracheobronchitis & MDR & $1.5 \mathrm{~g} / 8 \mathrm{~h}(13)$ & $S$ & E \\
\hline Zikri et al., 2019 [42] & Case report & 1 & $\mathrm{~F}, 14$ & Pneumonia & MDR & $1.5 \mathrm{~g} / 8 \mathrm{~h} \mathrm{(?)}$ & $S$ & N/D \\
\hline
\end{tabular}

$\mathrm{M}$, male; F, female; MDR, multidrug-resistant; $\mathrm{XDR}$, extensively drug-resistant, $\mathrm{PDR}$, pandrug-resistant; $\mathrm{CR}$, carbapenem-resistant; $\mathrm{S}$, success; F, failure; E, eradication; C, colonization; N/D, no data. 
Five countries were included: USA (12 studies [22,24,28-30,32-35,37,39,40]), Spain (4 studies [23,26,31,41]), Saudi Arabia (3 studies [21,25,42]), the UK (2 studies [36,38]), and France (1 study [27]).

The mean age was $58.89 \pm 19.33$ years (14-91 years). Sex was reported for 81 patients: males were $58(71.6 \%)$ and females were $23(28.4 \%)$.

The most common comorbidities were respiratory: cystic fibrosis was reported in 13 cases [24,29,35,37,39,40], while COPD was reported in 7 cases $[23,26,29,33,38,41]$. Ten patients had lungs transplanted $[26,27,29,39]$. The overall rate of respiratory failure was $17.5 \%$, and nine patients required tracheotomy before developing pneumonia [22,28,33,35,41].

Cardiac diseases were reported in 21 patients, and arterial hypertension was the most common (eight cases [21,23,25,39,41]). Seven patients had diabetes [23,25,26,37,39,42]. A renal impairment was reported in 10 cases: three patients presented with an acute kidney disease [26,32], while seven patients had a chronic kidney disease [21,25,35,37,39].

A history of solid cancer was described in seven patients [25,26,28,33,35,41]. Nine patients had blood cancer $[27,30,35,41]$, and three of them had undergone blood stem-cell transplant $[27,29,30]$.

Immunosupression conditions were reported in seven cases [26-28,31,41,42].

Neurological and abdominal comorbidities were also reported. The details are reported in Table 2.

Table 2. Comorbidities.

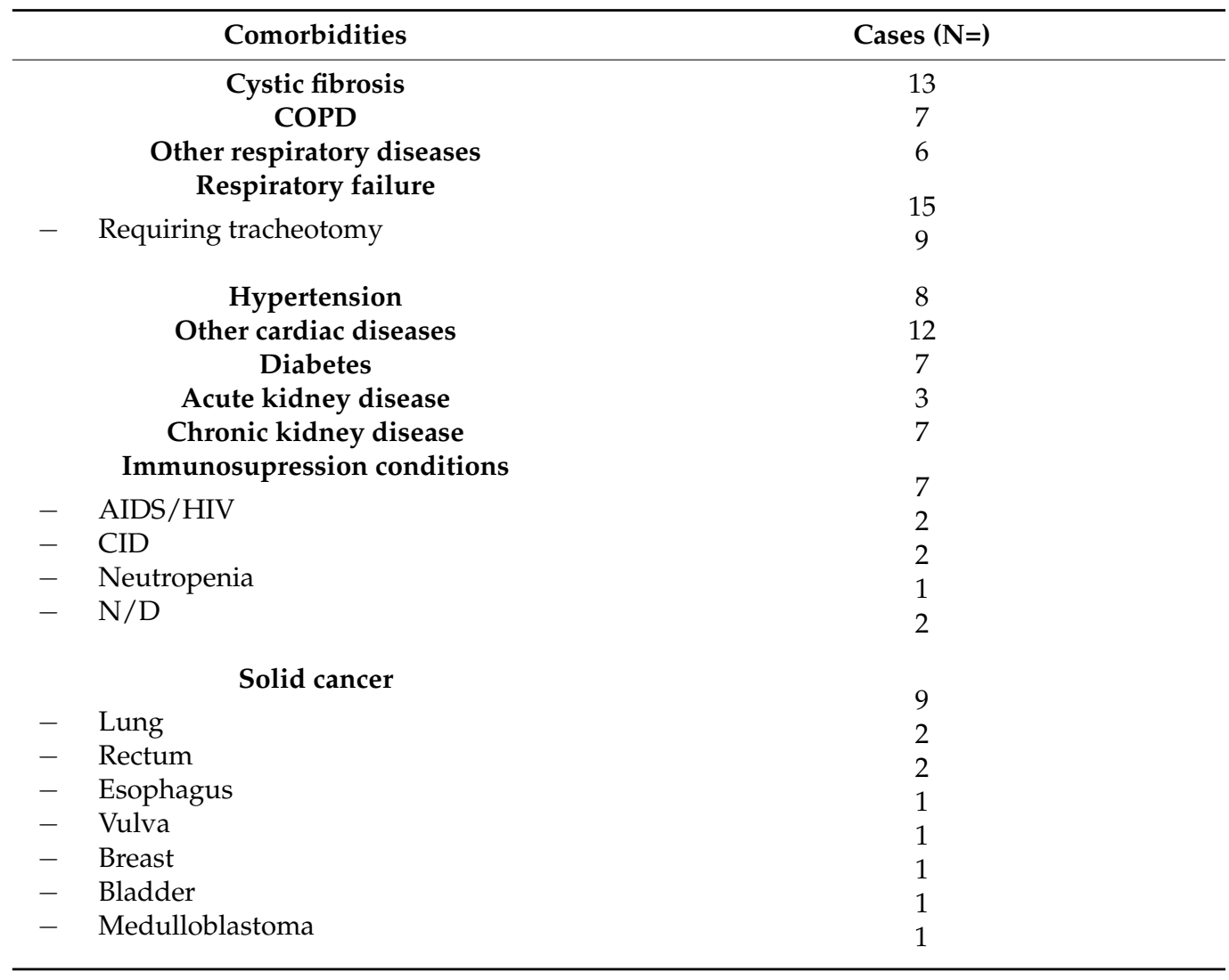


Table 2. Cont.

\begin{tabular}{|c|c|c|}
\hline & Comorbidities & Cases $(\mathrm{N}=)$ \\
\hline \multicolumn{3}{|c|}{ Blood cancer } \\
\hline- & Lymphoma & 3 \\
\hline- & Leukemia & $\begin{array}{l}3 \\
4\end{array}$ \\
\hline- & Myelodysplasia & 2 \\
\hline \multicolumn{3}{|c|}{ Transplantation } \\
\hline- & Lung & $\begin{array}{l}10 \\
10\end{array}$ \\
\hline- & Kidney & 3 \\
\hline- & Heart & 1 \\
\hline- & Liver & 1 \\
\hline & Dementia & 4 \\
\hline & Quadriplegia/tetraplegia & 5 \\
\hline & Other neurological diseases & 8 \\
\hline & Alcoholism/cirrhosis & 7 \\
\hline & Other gastrointestinal diseases & 4 \\
\hline
\end{tabular}

As shown in Figure 2, C/T was used to treat ventilator-associated pneumonia (VAP) in 14 cases [22,23,25,27,32,34], hospital-acquired pneumonia (HAP) in 11 cases [25,26,36,42], tracheobronchitis in 7 cases [29,31,41], and aspiration pneumonia in 1 case [21]. In the remaining 55 patients, the etiology was not clearly defined. Emphysema was associated in three cases $[29,35]$.

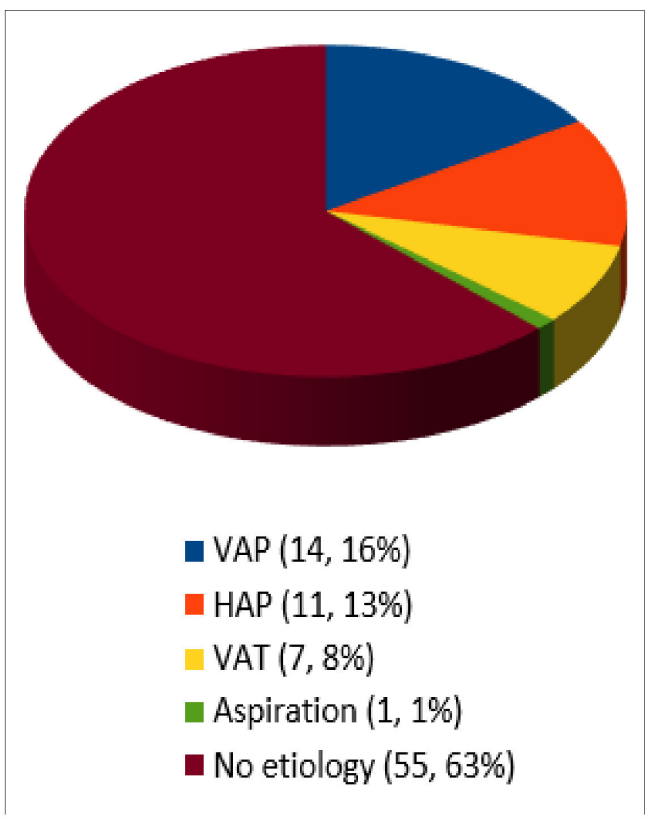

(a)

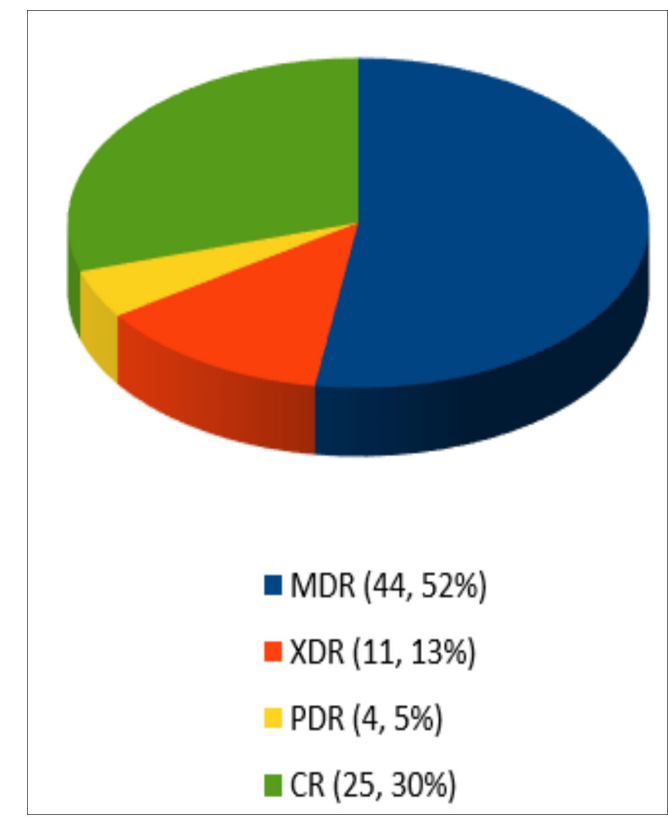

(b)

Figure 2. Type of infection (a) and resistance profile $(n, \%)(\mathbf{b})$.

MDR was reported in 44 cases [21,26,28-33,36,37,39-42], CR in 25 cases [25,34,35], XDR in 11 cases [27,41], PDR in 4 cases [22,23,38]. Among CR-PA, no cases of carbapenemaseproducing Enterobacteriaceae (CPE) were reported.

In 26 patients, a co-infection was detected (see Table 3). 
Table 3. Infection site and microorganisms involved.

\begin{tabular}{|c|c|c|}
\hline Author, Year & $\begin{array}{c}\text { Infection Site } \\
\text { (Biological Sample) }\end{array}$ & Microorganisms \\
\hline Alessa et al., 2018 & Lungs (N/D) & Pseudomonas Aeruginosa \\
\hline \multirow[t]{2}{*}{ Alqaid et al., 2015} & Urinary tract (culture) & Proteus mirabilis, Providencia stuartii \\
\hline & Lungs (BAS) & Pseudomonas Aeruginosa \\
\hline \multirow{2}{*}{ Álvarez Lerma et al., 2017} & Lungs (N/D) & Herpes simplex \\
\hline & Colon (N/D) & Clostridium difficile \\
\hline \multirow[t]{3}{*}{ Ang et al., 2016} & Blood (catheter culture) & Staphylococcus aureus \\
\hline & $\mathrm{N} / \mathrm{D}$ & Stenotrophomonas maltophilia \\
\hline & $\mathrm{N} / \mathrm{D}$ & Enterococcus faecalis \\
\hline \multirow[t]{3}{*}{ Dinh et al., 2017} & N/D & K. pneumoniae \\
\hline & $\mathrm{N} / \mathrm{D}$ & Citrobacter koseri \\
\hline & $\mathrm{N} / \mathrm{D}$ & P. aeruginosa \\
\hline \multirow[t]{4}{*}{ Gelfand et al., 2015} & $\mathrm{~N} / \mathrm{D}$ & Clostridium difficile \\
\hline & Lungs (N/D) & $M R S A$ \\
\hline & Lungs (N/D) & $M R S A$ \\
\hline & Lungs (N/D) & MRSA \\
\hline \multirow{5}{*}{ Haidar et al., 2018} & Lungs (N/D) & Serratia marcescens \\
\hline & Blood (N/D) & Vancomycin-resistant Enterococcus faecium \\
\hline & Blood $(\mathrm{N} / \mathrm{D})$ & Vancomycin-resistant Enterococcus faecium \\
\hline & Abdomen (wound culture) & Citrobacter fruendii \\
\hline & Blood $(\mathrm{N} / \mathrm{D})$ & Candida tropicalis \\
\hline Hernandez-Tejedor et al., 2016 & Blood (N/D) & Pseudomonas Aeruginosa \\
\hline \multirow{3}{*}{ Lewis et al., 2018} & Colon (N/D) & Clostridium difficile \\
\hline & Lungs (N/D) & Acinetobacter baumaunii \\
\hline & Blood (culture) & Staphylococcus epidermidis \\
\hline \multirow[t]{2}{*}{ Maniara et al., 2021} & Lungs (N/D) & Acinetobacter baumannii-calcoaceticus \\
\hline & Blood (culture) & Candida auris \\
\hline \multirow{2}{*}{ Munita et al., 2017} & Lungs (N/D) & K. pneumoniae \\
\hline & Lungs (N/D) & K. pneumoniae \\
\hline Romano et al., 2020 & $\mathrm{~N} / \mathrm{D}$ & MSSA, Pseudomonas aeruginosa \\
\hline Soliman et al., 2015 & Lungs (N/D) & MRSA, Pseudomonas aeruginosa \\
\hline \multirow{2}{*}{ Xipell et al., 2018} & Lungs (BAS) & MRSA \\
\hline & Lungs (BAS) & Enterobacter cloacae \\
\hline
\end{tabular}

MRSA, methicillin-resistant Staphylococcus aureus; MSSA, methicillin-sensitive Staphylococcus aureus; BAS, bronchial aspirate sample; $\mathrm{N} / \mathrm{D}$, no data.

As shown in Figure 3, out of 35 co-infection episodes, 17 microorganisms (49\%) were isolated in the lungs, 7 microorganisms (20\%) in the blood, 4 microorganisms (11\%) in the gastrointestinal tract, and 2 microorganisms $(6 \%)$ in the urinary tract. Of the five cases reported by Dinh et al., none of the sites of infection were reported.

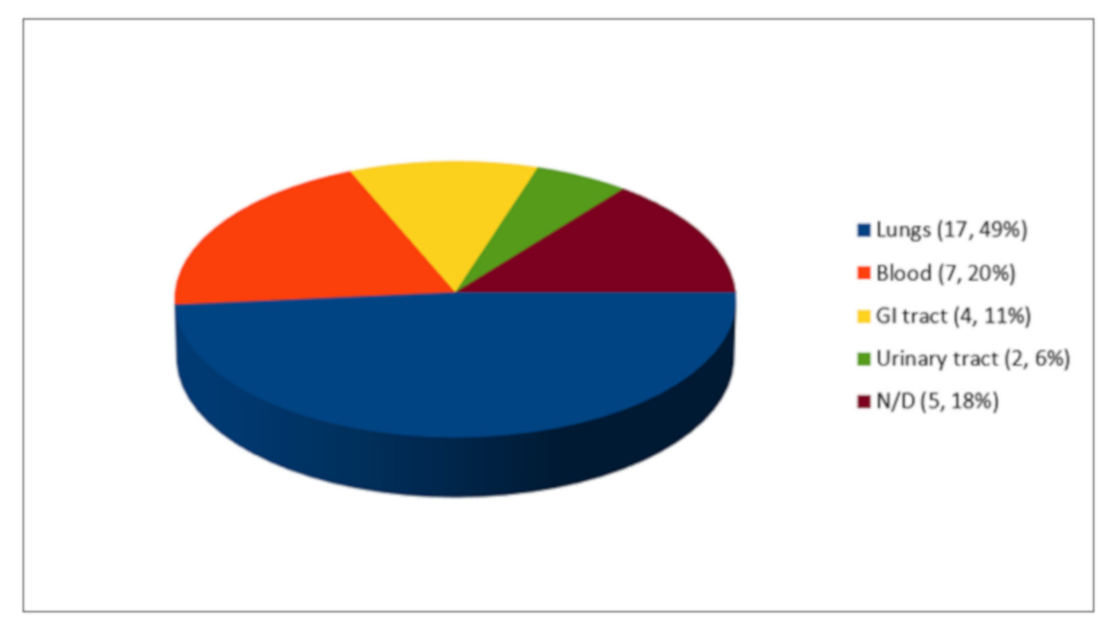

Figure 3. Co-infections $(n, \%)$. 
The most frequent bacterial microorganisms were Staphylococcus aureus (seven cases $[24,29,37,38,41])$, Pseudomonas aeruginosa (six cases [21,23,27,31,37,38]), Clostridium difficile (three cases $[23,28,33]$ ), and Klebsiella pneumoniae (three cases [27,35]). Two cases of fungal infections occurred due to Candida auris [34] and Candida tropicalis [29]. A case of herpes simplex pneumonia was reported by Álvarez Lerma et al. Details are shown in Figure 4 .

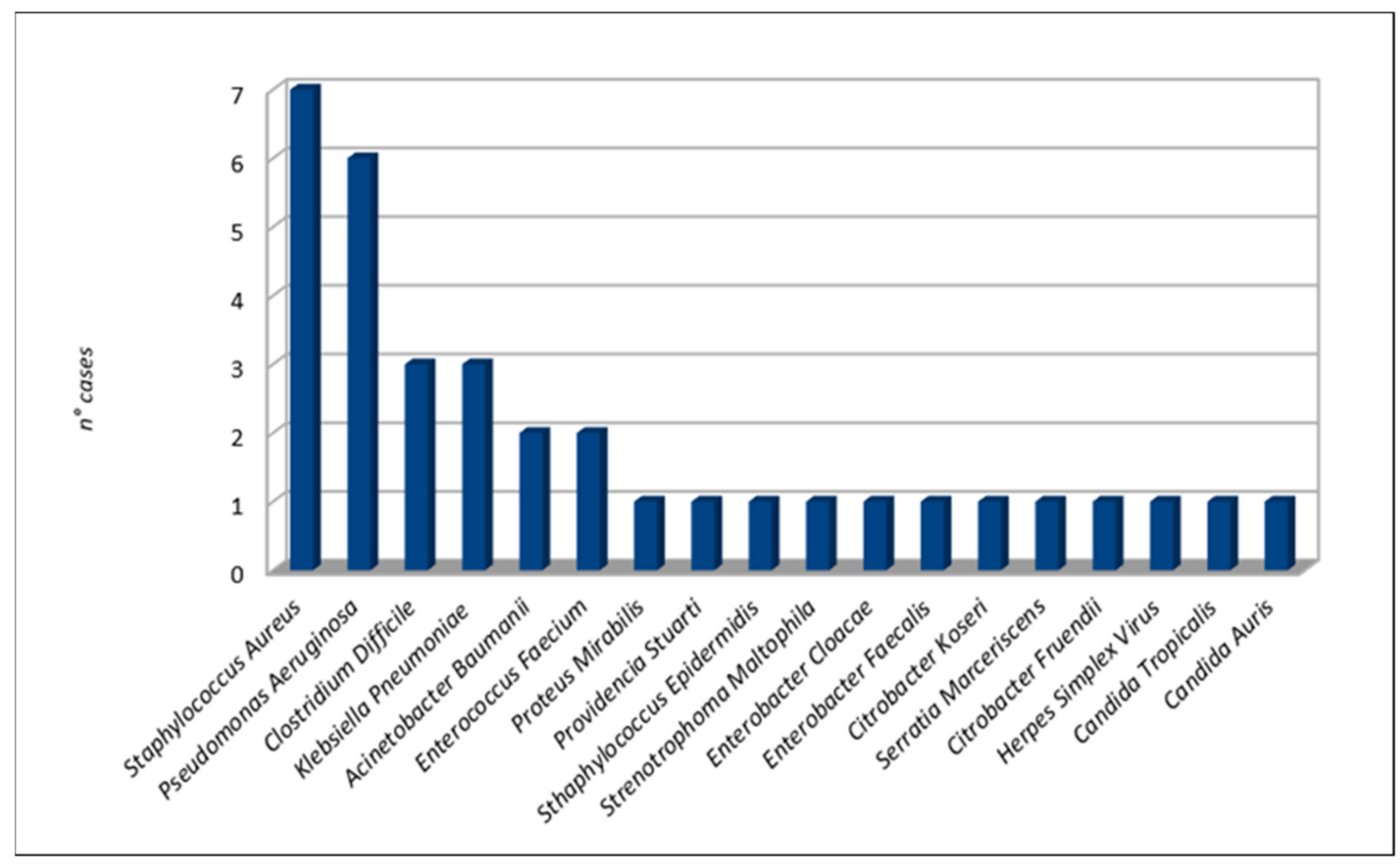

Figure 4. Co-infection microorganisms.

As shown in Table 1, empirical treatment was performed in 40 cases in the absence of microbiological isolation on the basis of clinical or radiological parameters. Targeted treatment consisted of $\mathrm{C} / \mathrm{T}$ alone or $\mathrm{C} / \mathrm{T}$ in association with other antibiotics. The most common association was with colistin intravenously injected or inhaled (27 patients [22,25-27,29,33-35]), tobramycin intravenously injected or inhaled (15 patients [21,29,33,35,40,41]), ciprofloxacin intravenously injected (7 patients $[24,29,37,40])$, amikacin intravenously injected (5 patients $[27,41,42])$, and linezolid intravenously injected (4 patients [29,38]).

In 31 cases [22,25-27,29,31,33,35,36,41,42], CT was administered as $1.5 \mathrm{~g}$ every $8 \mathrm{~h}$, while in 26 cases as 3 g every $8 \mathrm{~h}[26-30,32,34,35,37,38,40]$. Other $\mathrm{C} / \mathrm{T}$ regimes were 375 $\mathrm{mg}$ every $8 \mathrm{~h}$ (seven patients [29,35]), $750 \mathrm{mg}$ every $8 \mathrm{~h}$ (six patients [23,35]), $150 \mathrm{mg}$ every $8 \mathrm{~h}$ (two patients [29]), $2 \mathrm{~g}$ every $8 \mathrm{~h}$ (one patient [26]), and $3 \mathrm{~g}$ every $12 \mathrm{~h}$ (one patient [39]). In Alessa et al., a bolus ( $1.5 \mathrm{~g}$ ) was performed before starting C/T $300 \mathrm{mg}$ every $8 \mathrm{~h}$. In three cases $[23,24,41]$, the administration protocol was adapted during the treatment. Three studies (six patients $[25,27,41]$ ) did not report the $\mathrm{C} / \mathrm{T}$ regime.

$\mathrm{C} / \mathrm{T}$ mean duration therapy was $15.61 \pm 13.28$ days; it ranged from 3 days $[26,29]$ to 103 days [30].

Only six patients presented adverse events, but this did not lead to the therapy discontinuation. The reported AEs were mild transaminasis [24], confusion/hallucinations, renal failure, anemia [27], leukocytosis [34], eosinophilia/eosinophyluria, and interstitial nephritis (Munita et al.). 
As shown in Figure 5, the overall success rate of $\mathrm{C} / \mathrm{T}$ was $72.6 \%$ (61/84 cases). Failure was more frequent when considering CR-PA ( $40 \%, 10 / 25$ cases), followed by XDR-PA (36.4\%, 4/11 cases) and MDR-PA (20.5\%, 9/44 cases). Exitus occurred in 13 cases.

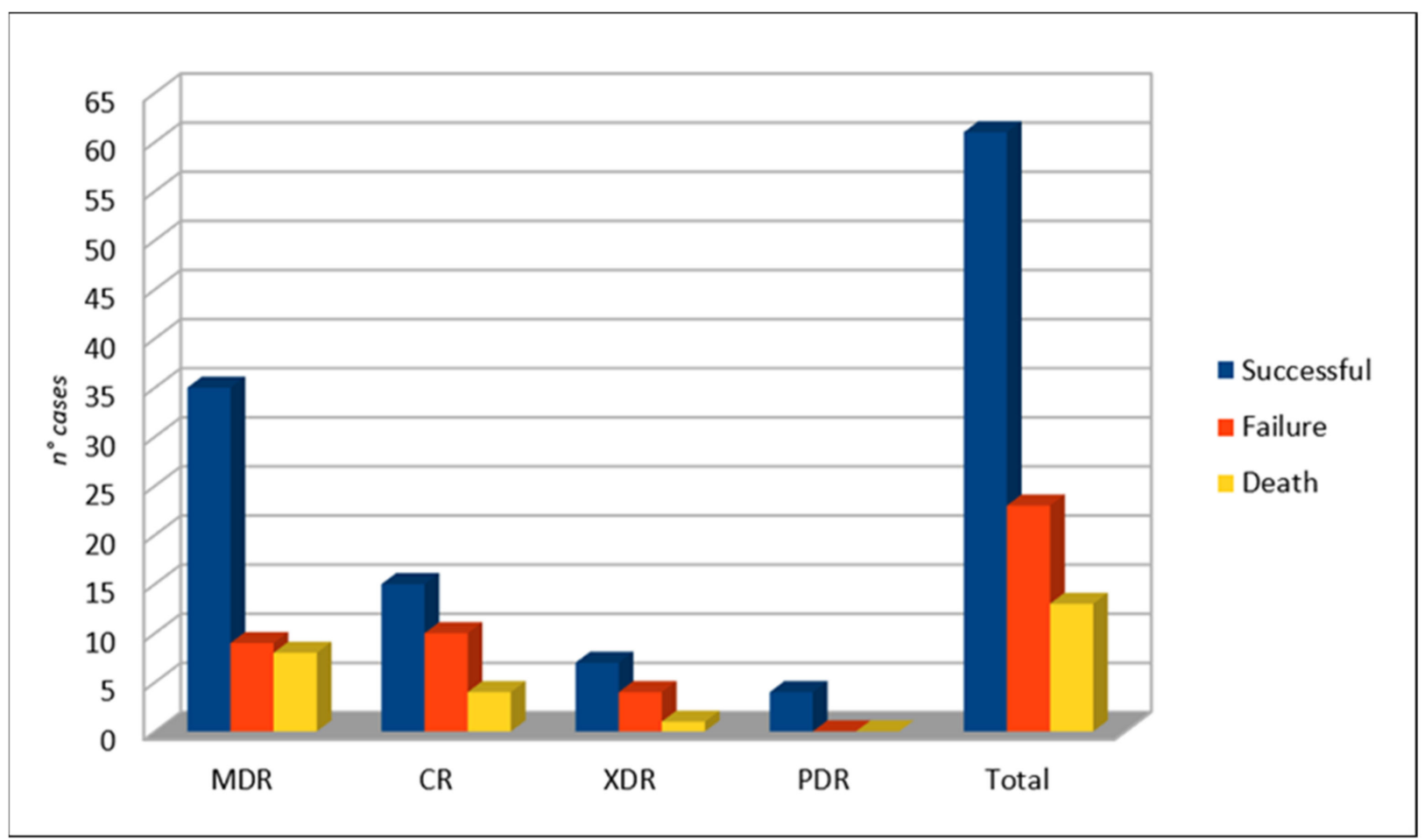

Figure 5. Clinical outcome.

After C/T therapy, the eradication of infection occurred in 26 patients, whereas the patients colonized by PA were 22 in number (see Figure 6). In 36 cases, no microbiological outcome data were available.

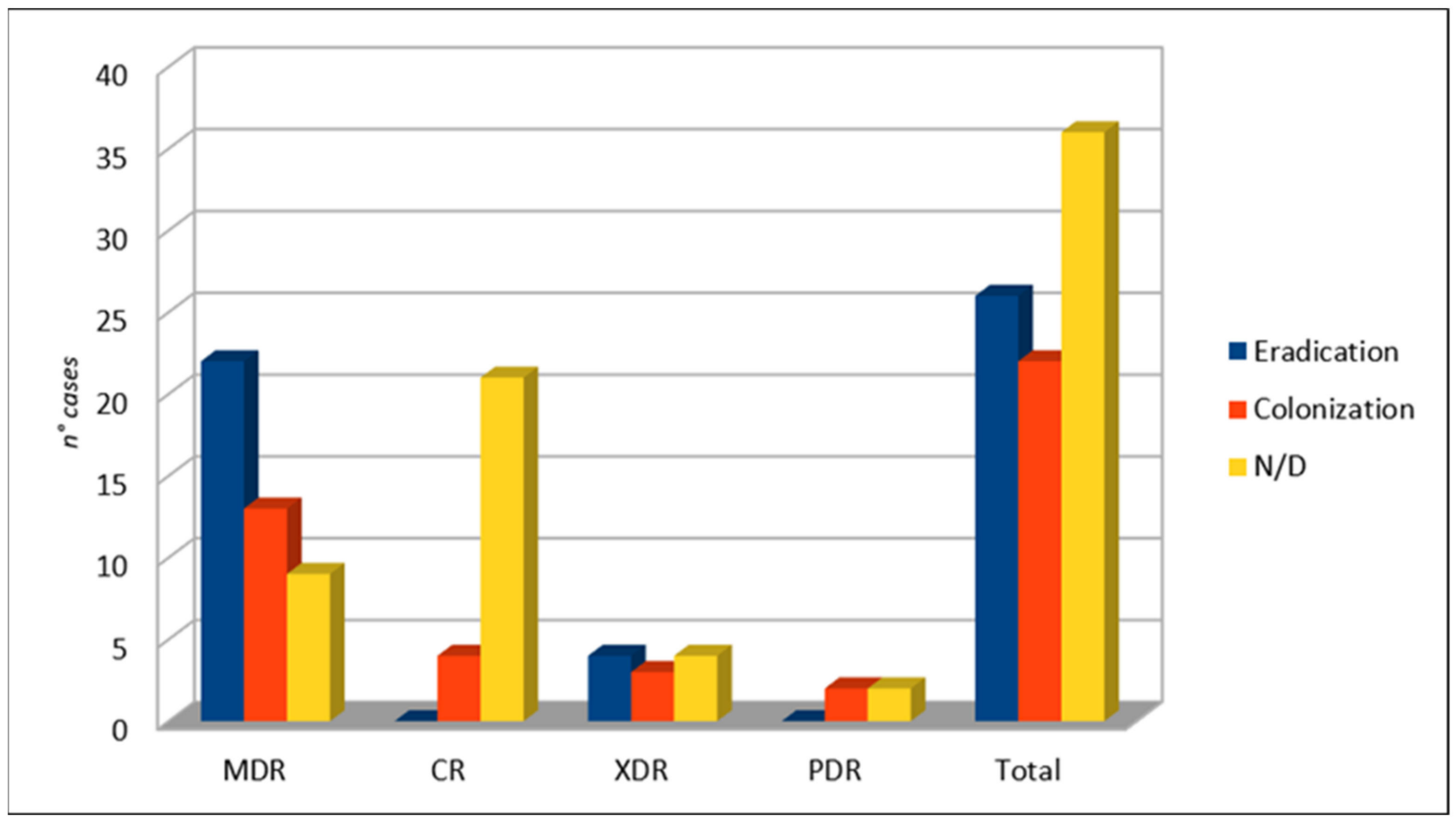

Figure 6. Microbiological outcome. 


\section{Discussion}

PA is a challenging Gram-negative bacterium due to its intrinsic and acquired resistance mechanisms and its ability to develop biofilms [13]. To date, the optimal management of the PA infections, especially in cases of multidrug-resistant strains, it is not clarified. First, the superiority of combination therapy over monotherapy as definitive treatment is not established [43]. Current recommendations suggest double anti-PA empirical therapy, especially if resistant isolates are suspected [44,45]. Second, there is no agreement on the antibiotic treatment to choose. $\mathrm{C} / \mathrm{T}$ is emerging as a good option among $\beta$-lactams alone or in combination (especially with colistin or amikacin) for acute invasive PA infections $[14,45]$. In vitro, $C / T$ is the most active $\beta$-lactam against $P A$, even in the case of resistant strains. C/T is active against $75-89 \%$ of the isolates not sensitive to ceftazidime, piperacillin/tazobactam, and meropenem [46].

In our systematic review, the clinical success rate was $72.6 \%$ across a wide range of comorbidities. According to the literature, PA causes infections in patients with comorbidities, such as cystic fibrosis, chronic structural lung disease (e.g., bronchiectasis, COPD), impaired immune defenses (e.g., HIV infection, malignancy with neutropenia or recent chemotherapy, organ transplant recipients), diabetes mellitus, renal failure and hemodialysis, and chronic cardiovascular or neurological disease [47]. Notably, clinical success was even higher when considering MDR-PA cases only (83.6\%). According to previous data, CR-PA represents a therapeutic problem with a failure rate of $40 \%$ in present studies. In this direction, the WHO supports the development of new antibiotics that are active against multidrug-resistant Gram-negative bacteria [15]. The $45.8 \%$ of patients treated with C/T presented colonization by PA.

The most common therapeutic regimens were $1.5 \mathrm{~g}$ every $8 \mathrm{~h}$ and $3 \mathrm{~g}$ every $8 \mathrm{~h}$. The latter represents the recommended dosage regimen for treating HAP and VAP [11,12]. Ceftolozane has shown adequate penetration in lung tissue, close to $50 \%$, with $2 \mathrm{~g}$ doses in an extended infusion of $3 \mathrm{~h}$ [48].

Importantly, C/T was well tolerated. Only six patients presented adverse events, but no one had to stop the treatment. The increased dose of $\mathrm{C} / \mathrm{T}$ could be associated with an increase in the occurrence of adverse effects, such as elevation of liver enzymes, hypotension, or cutaneous reactions [48].

This study contributes to the accumulation of so-called "real world data", which, when properly interpreted, can provide suggestions and guidelines [49]. Indeed, due to the recognized gap between the global burden of resistance bacteria and new antibiotics under development, many proposals have been made to optimize the design of clinical trials, but not all recommendations are feasible or immediately applicable [50,51]. In this way, real-world evidence may come as useful.

To summarize, this is the first systematic review of the use of $C / T$ in resistant pulmonary PA infections. Its strength is based on rigorous inclusion criteria, which allow it to collect data exclusively on MDR-PA, XDR-PA, PDR-PA, and CR-PA infections and determine the outcome.

Limitations. This study presents some limitations. First, it was based on observational studies, the majority of which were case series or case reports. Due to the greater potential for bias, they are often excluded from systematic reviews of treatments. In this report, case series and observational studies contribute substantially to the available evidence base, and their results supplement the limited evidence available from other studies. Second, a meta-analysis was not performed, owing to the design of most of the studies (case report, case series) and the lack of a comparator.

\section{Conclusions}

C/T may be a valid therapeutic option to treat MDR-PA, XDR-PA, PDR-PA, and CR-PA infections. However, further data are necessary to define its optimal treatment dosage and duration. 
Author Contributions: Conceptualization, L.G.G. and P.S.; methodology, M.B.P. and F.G.; software, M.B.P.; validation, L.G.G., M.C.P. and P.S.; formal analysis, L.G.G., M.C.P. and P.S.; investigation, L.G.G., M.C.P. and P.S.; resources, L.G.G., M.C.P. and P.S.; data curation, L.G.G., M.C.P. and P.S.; writing-original draft preparation, L.G.G. and M.C.P.; writing-review and editing, L.G.G., M.C.P. and P.S.; visualization, M.B.P. and F.G.; supervision, C.A. and P.S.; project administration, L.G.G. and P.S. All authors have read and agreed to the published version of the manuscript.

Funding: This research received no external funding.

Institutional Review Board Statement: Not applicable.

Informed Consent Statement: Not applicable.

Data Availability Statement: Data were collected in the Department of Women, Child, General and Specialistic Surgery, University of Campania “L. Vanvitelli”, Naples, Italy.

Conflicts of Interest: The authors declare no conflict of interest.

\section{References}

1. Gentile, I.; Maraolo, A.E.; Borgia, G. What is the role of the new $\beta$-lactam/ $\beta$-lactamase inhibitors ceftolozane/tazobactam and ceftazidime/avibactam? Expert Rev. Anti-Infect. Ther. 2016, 14, 875-878. [CrossRef] [PubMed]

2. US Food and Drug Administration (FDA). Zerbaxa (ceftolozane/tazobactam) Letter of Approval. Available online: https: //www.accessdata.fda.gov/drugsatfda_docs/nda/014/206829Orig1s000Approv.pdf (accessed on 11 April 2021).

3. European Medicines Agency (EMA). Zerbaxa (ceftolozane/tazobactam) Letter of Approval. Available online: https://www.ema. europa.eu/en/medicines/human/EPAR/zerbaxa\#authorisation-details-section (accessed on 11 April 2021).

4. Solomkin, J.; Hershberger, E.; Miller, B.; Popejoy, M.; Friedland, I.; Steenbergen, J.; Yoon, M.; Collins, S.; Yuan, G.; Barie, P.S.; et al. Ceftolozane/Tazobactam Plus Metronidazole for Complicated Intra-abdominal Infections in an Era of Multidrug Resistance: Results From a Randomized, Double-Blind, Phase 3 Trial (ASPECT-cIAI). Clin. Infect. Dis. 2015, 60, 1462-1471. [CrossRef] [PubMed]

5. Wagenlehner, F.M.; Umeh, O.; Steenbergen, J.; Yuan, G.; Darouiche, R.O. Ceftolozane-tazobactam compared with levofloxacin in the treatment of complicated urinary-tract infections, including pyelonephritis: A randomised, double-blind, phase 3 trial (ASPECT-cUTI). Lancet 2015, 385, 1949-1956. [CrossRef]

6. Karaiskos, I.; Lagou, S.; Pontikis, K.; Rapti, V.; Poulakou, G. The "Old" and the "New" Antibiotics for MDR Gram-Negative Pathogens: For Whom, When, and How. Front Public Health 2019, 7, 151. [CrossRef]

7. Walter, J.; Haller, S.; Quinten, C.; Kärki, T.; Zacher, B.; Eckmanns, T.; Abu Sin, M.; Plachouras, D.; Kinross, P.; Suetens, C. Healthcare-associated pneumonia in acute care hospitals in European Union/European Economic Area countries: An analysis of data from a point prevalence survey, 2011 to 2012. Eurosurveillance 2018, 23, 1700843. [CrossRef]

8. Kollef, M.H.; Chastre, J.; Fagon, J.-Y.; François, B.; Niederman, M.S.; Rello, J.; Torres, A.; Vincent, J.-L.; Wunderink, R.G.; Go, K.W.; et al. Global Prospective Epidemiologic and Surveillance Study of Ventilator-Associated Pneumonia due to Pseudomonas aeruginosa*. Crit. Care Med. 2014, 42, 2178-2187. [CrossRef]

9. Kalil, A.C.; Metersky, M.L.; Klompas, M.; Muscedere, J.; Sweeney, D.A.; Palmer, L.B.; Napolitano, L.M.; O'Grady, N.P.; Bartlett, J.G.; Carratalà, J.; et al. Management of Adults With Hospital-acquired and Ventilator-associated Pneumonia: 2016 Clinical Practice Guidelines by the Infectious Diseases Society of America and the American Thoracic Society. Clin. Infect. Dis. 2016, 63, e61-e111. [CrossRef]

10. Torres, A.; Niederman, M.S.; Chastre, J.; Ewig, S.; Fernandez-Vandellos, P.; Hanberger, H.; Kollef, M.; Li Bassi, G.; Luna, C.M.; Martin-Loeches, I.; et al. International ERS/ESICM/ESCMID/ALAT guidelines for the management of hospital-acquired pneumonia and ventilator-associated pneumonia: Guidelines for the management of hospital-acquired pneumonia (HAP)/ventilatorassociated pneumonia (VAP) of the European Respiratory Society (ERS), European Society of Intensive Care Medicine (ESICM), European Society of Clinical Microbiology and Infectious Diseases (ESCMID) and Asociación Latinoamericana del Tórax (ALAT). Eur. Respir. J. 2017, 50, 1700582.

11. US Food and Drug Administration (FDA). FDA Approves New Treatment for Hospital-Acquired and Ventilator-Associated Bacterial Pneumonia; FDA: Atlanta, GA, USA, 2019. Available online: https://www.fda.gov/news-events/press-announcements/fdaapproves-new-treatment-hospital-acquired-and-ventilator-associated-bacterial-pneumonia (accessed on 11 April 2021).

12. European Medicines Agency (EMA). Zerbaxa. EMA. 2019. Available online: https://www.ema.europa.eu/en/medicines/ human/EPAR/zerbaxa (accessed on 11 April 2021).

13. Moradali, M.F.; Ghods, S.; Rehm, B.H.A. Pseudomonas aeruginosa Lifestyle: A Paradigm for Adaptation, Survival, and Persistence. Front. Cell. Infect. Microbiol. 2017, 7, 39. [CrossRef]

14. Giani, T.; Arena, F.; Pollini, S.; Di Pilato, V.; D’Andrea, M.M.; De Angelis, L.H.; Bassetti, M.; Rossolini, G.M.; Vismara, C.; Luzzaro, F.; et al. Italian nationwide survey on Pseudomonas aeruginosa from invasive infections: Activity of ceftolozane/tazobactam and comparators, and molecular epidemiology of carbapenemase producers. J. Antimicrob. Chemother. 2018, 73, 664-671. [CrossRef] 
15. Tacconelli, E.; Carrara, E.; Savoldi, A.; Harbarth, S.; Mendelson, M.; Monnet, D.L.; Pulcini, C.; Kahlmeter, G.; Kluytmans, J.; Carmeli, Y.; et al. Discovery, research, and development of new antibiotics: The WHO priority list of antibiotic-resistant bacteria and tuberculosis. Lancet Infect. Dis. 2018, 18, 318-327. [CrossRef]

16. Zhanel, G.G.; Lawson, C.D.; Adam, H.; Schweizer, F.; Zelenitsky, S.; Lagacé-Wiens, P.R.; Denisuik, A.; Rubinstein, E.; Gin, A.S.; Hoban, D.J.; et al. Ceftazidime-avibactam: A novel cephalosporin/b-lactamase inhibitor combination. Drugs 2013, 73, 159-177. [CrossRef]

17. Sader, H.S.; Rhomberg, P.R.; Farrell, D.J.; Jones, R.N. Antimicrobial Activity of CXA-101, a Novel Cephalosporin Tested in Combination with Tazobactam against Enterobacteriaceae, Pseudomonas aeruginosa, and Bacteroides fragilis Strains Having Various Resistance Phenotypes. Antimicrob. Agents Chemother. 2011, 55, 2390-2394. [CrossRef] [PubMed]

18. Farrell, D.J.; Flamm, R.K.; Sader, H.S.; Jones, R.N. Antimicrobial Activity of Ceftolozane-Tazobactam Tested against Enterobacteriaceae and Pseudomonas aeruginosa with Various Resistance Patterns Isolated in U.S. Hospitals (2011-2012). Antimicrob. Agents Chemother. 2013, 57, 6305-6310. [CrossRef]

19. Moher, D.; Liberati, A.; Tetzlaff, J.; Altman, D.G. The PRISMA Group. Preferred Reporting Items for Systematic Reviews andMeta-Analyses: The PRISMA Statement. BMJ 2009, 339, b2535. [CrossRef] [PubMed]

20. Magiorakos, A.-P.; Srinivasan, A.; Carey, R.B.; Carmeli, Y.; Falagas, M.E.; Giske, C.G.; Harbarth, S.; Hindler, J.F.; Kahlmeter, G.; Olsson-Liljequist, B.; et al. Multidrug-resistant, extensively drug-resistant and pandrug-resistant bacteria: An international expert proposal for interim standard definitions for acquired resistance. Clin. Microbiol. Infect. 2012, 18, 268-281. [CrossRef] [PubMed]

21. Alessa, M.A.; Almangour, T.A.; Alhossan, A.; Alkholief, M.A.; Alhokail, M.; Tabb, D.E. Ceftolozane-tazobactam for the treatment of multidrug-resistant Pseudomonas aeruginosa pneumonia in a patient receiving intermittent hemodialysis. Am. J. Health Syst. Pharm. 2018, 75, e184-e188. [CrossRef]

22. Alqaid, A.; Dougherty, C.; Ahmad, S. Triple antibiotic therapy with ceftolozane/tazobactam, colistin and rifampin for panresistant Pseudomonas aeruginosa ventilator-associated pneumonia. Southwest Respir. Crit. Care Chron. 2015, 3. [CrossRef]

23. Sorli, L.; Recasens, L.; García, M.M. Ceftolozane-tazobactam for the treatment of ventilator-associated infections by colistinresistant Pseudomonas aeruginosa. Rev. Esp. Quimioter. 2017, 30, 224-228.

24. Ang, J.Y.; Abdel-Haq, N.; Zhu, F.; Thabit, A.K.; Nicolau, D.P.; Satlin, M.J.; Van Duin, D. Multidrug-Resistant Pseudomonas aeruginosa Infection in a Child with Cystic Fibrosis. Antimicrob. Agents Chemother. 2016, 60, 5627-5630. [CrossRef]

25. Bosaeed, M.; Ahmad, A.; Alali, A.; Mahmoud, E.; Alswidan, L.; Alsaedy, A.; Aljuhani, S.; Alalwan, B.; Alshamrani, M.; Alothman, A. Experience With Ceftolozane-Tazobactam for the Treatment of Serious Pseudomonas aeruginosa Infections in Saudi Tertiary Care Center. Infect. Dis. 2020, 13. [CrossRef] [PubMed]

26. Castón, J.J.; De La Torre, Á.; Ruiz-Camps, I.; Sorlí, M.L.; Torres, V.; Torre-Cisneros, J. Salvage Therapy with CeftolozaneTazobactam for Multidrug-Resistant Pseudomonas aeruginosa Infections. Antimicrob. Agents Chemother. 2017, 61, e02136-16. [CrossRef] [PubMed]

27. Dinh, A.; Wyplosz, B.; Kernéis, S.; Lebeaux, D.; Bouchand, F.; Duran, C.; Béraud, G.; Lazaro, P.; Davido, B.; Hénard, S.; et al. Use of ceftolozane/tazobactam as salvage therapy for infections due to extensively drug-resistant Pseudomonas aeruginosa. Int. J. Antimicrob. Agents 2017, 49, 782-783. [CrossRef] [PubMed]

28. Gelfand, M.S.; Cleveland, K.O. Ceftolozane/Tazobactam Therapy of Respiratory Infections due to Multidrug-Resistant Pseudomonas aeruginosa. Clin. Infect. Dis. 2015, 61, 853-855. [CrossRef] [PubMed]

29. Haidar, G.; Philips, N.J.; Shields, R.K.; Snyder, D.; Cheng, S.; Potoski, B.A.; Doi, Y.; Hao, B.; Press, E.G.; Cooper, V.S.; et al. Ceftolozane-Tazobactam for the Treatment of Multidrug-Resistant Pseudomonas aeruginosa Infections: Clinical Effectiveness and Evolution of Resistance. Clin. Infect. Dis. 2017, 65, 110-120. [CrossRef] [PubMed]

30. Hakki, M.; Lewis, J.S. Ceftolozane-tazobactam therapy for multidrug-resistant Pseudomonas aeruginosa infections in patients with hematologic malignancies and hematopoietic-cell transplant recipients. Infection 2018, 46, 431-434. [CrossRef] [PubMed]

31. Hernández-Tejedor, A.; Merino-Vega, C.D.; Martín-Vivas, A.; de Luna-González, R.R.; Delgado-Iribarren, A.; Gabán-Díez, Á.; Temprano-Gómez, I.; de la Calle-Pedrosa, N.; González-Jiménez, A.I.; Algora-Weber, A. Successful treatment of multidrugresistant Pseudomonas aeruginosa breakthrough bacteremia with ceftolozane/tazobactam. Infection 2017, 45, 115-117. [CrossRef] [PubMed]

32. Kuti, J.L.; Ghazi, I.M.; Quintiliani, R.; Shore, E.; Nicolau, D.P. Treatment of multidrug-resistant Pseudomonas aeruginosa with ceftolozane/tazobactam in a critically ill patient receiving continuous venovenous haemodiafiltration. Int. J. Antimicrob. Agents 2016, 48, 342-343. [CrossRef]

33. Lewis, P.O.; Cluck, D.; Tharp, J.L.; Krolikowski, M.A.; Patel, P.D. Failure of ceftolozane-tazobactam salvage therapy in complicated pneumonia with lung abscess. Clin. Case Rep. 2018, 6, 1308-1312. [CrossRef]

34. Maniara, B.P.; Wells, I. Ceftolozane/Tazobactam-Induced Leukocytosis and Clinical Failure in a Patient Being Treated for Ventilator-Associated Pneumonia Caused by Carbapenem-Resistant Pseudomonas aeruginosa: A Case Report. SN Compr. Clin. Med. 2021, 3, 701-704. [CrossRef]

35. Munita, J.M.; Aitken, S.L.; Miller, W.R.; Perez, F.; Rosa, R.; A Shimose, L.; Lichtenberger, P.N.; Abbo, L.M.; Jain, R.; Nigo, M.; et al. Multicenter Evaluation of Ceftolozane/Tazobactam for Serious Infections Caused by Carbapenem-Resistant Pseudomonas aeruginosa. Clin. Infect. Dis. 2017, 65, 158-161. [CrossRef]

36. Plant, A.J.; Dunn, A.; Porter, R.J. Ceftolozane-tazobactam resistance induced in vivo during the treatment of MDR Pseudomonas aeruginosa pneumonia. Expert Rev. Anti-Infect. Ther. 2018, 16, 367-368. [CrossRef] [PubMed] 
37. Romano, M.T.; Premraj, S.; Bray, J.M.; Murillo, L.C. Ceftolozane/tazobactam for pulmonary exacerbation in a 63-year-old cystic fibrosis patient with renal insufficiency and an elevated MIC to Pseudomonas aeruginosa. IDCases 2020, 21, e00830. [CrossRef] [PubMed]

38. Soliman, R.; Woodford, N.; Pike, R.; Livermore, D.; Lynch, S.; Turton, J.; Meader, E.; Hill, R. Successful ceftolozane/tazobactam treatment of chronic pulmonary infection with pan-resistant Pseudomonas aeruginosa. JMM Case Reports 2015, 2. [CrossRef]

39. Stokem, K.; Zuckerman, J.B.; Nicolau, D.P.; Wungwattana, M.; Sears, E.H. Use of ceftolozane-tazobactam in a cystic fibrosis patient with multidrug-resistant pseudomonas infection and renal insufficiency. Respir. Med. Case Rep. 2018, 23, 8-9. [CrossRef]

40. Vickery, S.B.; McClain, D.; Wargo, K.A. Successful Use of Ceftolozane-Tazobactam to Treat a Pulmonary Exacerbation of Cystic Fibrosis Caused by Multidrug-ResistantPseudomonas aeruginosa. Pharmacotherapy 2016, 36, e154-e159. [CrossRef]

41. Xipell, M.; Paredes, S.; Fresco, L.; Bodro, M.; Marco, F.; Martínez, J.; Soriano, A. Clinical experience with ceftolozane/tazobactam in patients with serious infections due to resistant Pseudomonas aeruginosa. J. Glob. Antimicrob. Resist. 2018, 13, 165-170. [CrossRef] [PubMed]

42. Zikri, A.; El Masri, K. Use of Ceftolozane/tazobactam for the Treatment of Multidrug-resistant Pseudomonas aeruginosa Pneumonia in a Pediatric Patient with Combined Immunodeficiency (CID): A Case Report from a Tertiary Hospital in Saudi Arabia. Antibiotiotics 2019, 8, 67. [CrossRef]

43. Paul, M.; Leibovici, L. Editorial Commentary: Combination Therapy for Pseudomonas aeruginosa Bacteremia: Where Do We Stand? Clin. Infect. Dis. 2013, 57, 217-220. [CrossRef]

44. Bassetti, M.; Vena, A.; Russo, A.; Croxatto, A.; Calandra, T.; Guery, B. Rational approach in the management of Pseudomonas aeruginosa infections. Curr. Opin. Infect. Dis. 2018, 31, 578-586. [CrossRef]

45. Mensa, J.; Barberán, J.; Soriano, A.; Llinares, P.; Marco, F.; Cantón, R.; Bou, G.; del Castillo, J.G.; Maseda, E.; Azanza, J.R.; et al. Antibiotic selection in the treatment of acute invasive infections by Pseudomonas aeruginosa: Guidelines by the Spanish Society of Chemotherapy. Rev. Esp. Quimioter. 2018, 31, 78-100. [PubMed]

46. Lob, S.H.; Hoban, D.J.; Young, K.; Motyl, M.R.; Sahm, D.F. Activity of ceftolozane-tazobactam and comparators against Pseudomonas aeruginosa from patients in different risk strata-SMART United States 2016-2017. J. Glob. Antimicrob. Resist. 2020, 20, 209-213. [CrossRef] [PubMed]

47. Lynch, J.P., 3rd; Zhanel, G.G.; Clark, N.M. Emergence of Antimicrobial Resistance among Pseudomonas aeruginosa: Implications for Therapy. Semin. Respir. Crit. Care Med. 2017, 38, 326-345. [PubMed]

48. Ruiz, J.; Ferrada, A.; Salavert, M.; Gordon, M.; Villarreal, E.; Castellanos-Ortega, Á.; Ramirez, P. Ceftolozane/Tazobactam Dosing Requirements Against Pseudomonas aeruginosa Bacteremia. Dose Response 2020, 18, 1559325819885790. [CrossRef] [PubMed]

49. Blonde, L.; Khunti, K.; Harris, S.B.; Meizinger, C.; Skolnik, N.S. Interpretation and impact of real-world clinical data for the practicing clinician. Adv. Ther. 2018, 35, 1763-1774. [CrossRef]

50. De Kraker, M.E.A.; Sommer, H.; de Velde, F.; Gravestock, I.; Weiss, E.; McAleenan, A.; Nikolakopoulos, S.; Amit, O.; Ashton, T.; Beyersmann, J.; et al. Optimizing the design and analysis of clinical trials for antibacteri- als against multidrug-resistant organisms: A white paper from COMBACTE's STAT-Net. Clin. Infect. Dis. 2018, 67, 1922-1931.

51. Paul, M.; Scudeller, L. Clinical research designs to study treatment effects for multidrug-resistant bacteria. Clin. Microbiol. Infect. 2019, 25, 929-931. [CrossRef] 